\title{
Performance Enhancement Schemes of OFDMA System for Broadband Wireless Access
}

\author{
Dong-Hyun Park, So-Young Yeo, Jee-Hoon Kim, Young-Hwan You, \\ and Hyoung-Kyu Song \\ uT Communication Research Institute, Sejong University, Seoul, Korea \\ mith80@sdc.sejong.ac.kr, yeossoh@sdc.sejong.ac.kr, bid4u@sdc.sejong.ac.kr, \\ yhyou@sejong.ac.kr, songhk@sejong.ac.kr
}

\begin{abstract}
Orthogonal frequency-division multiple access (OFDMA), which is a combination of orthogonal frequency-division multiplexing (OFDM) with frequency-division multiple access (FDMA), is regarded as a promising solution for enhancing the performance of interactive wireless systems in ubiquitous mobile communication environment. In such an application, this paper presents an investigation into improving the channel estimation scheme and of the effects of symbol timing misalignment when OFDMA is used as an access scheme. Under OFDMA uplink channel environments, appropriate symbol length of CAZAC sequences as a preamble could be utilized in accordance with the number of transmitting antenna and channel condition. The effect of the number of CAZAC sequences for channel estimation is also presented in terms of mean square error (MSE). Taking into account the effect of multiple access interference (MAI) introduced by a symbol timing misalignment, the symbol error rate (BER) and throughput performance are investigated for a typical OFDMA uplink scenario.
\end{abstract}

Keywords: OFDMA, channel estimation, MAI, CAZAC.

\section{Introduction}

Mobile users are demanding anywhere and anytime access to high-speed data real-and non-real time multimedia services from next-generation wireless systems. In accordance with the requirements of users, the future generations of broadband wireless communications will provide to subscribers the high quality of service (QoS) and bit rates by employing a variety of techniques. Also, they will support the future ubiquitous communications systems. In wireless multi-user environments, one of the reliable solutions for such communication systems is OFDMA technology. OFDMA, also referred to as Multiuser-OFDM, is being considered as a modulation and multiple access method for 4-th generation wireless networks. OFDMA is an extension of Orthogonal Frequency Division Multiplexing (OFDM), which is currently the modulation of choice for high speed data access systems such as IEEE 802.11a/g wireless LAN (WiFi) and IEEE 802.16a/d/e wireless broadband access systems (WiMAX) [1]-[3]. 


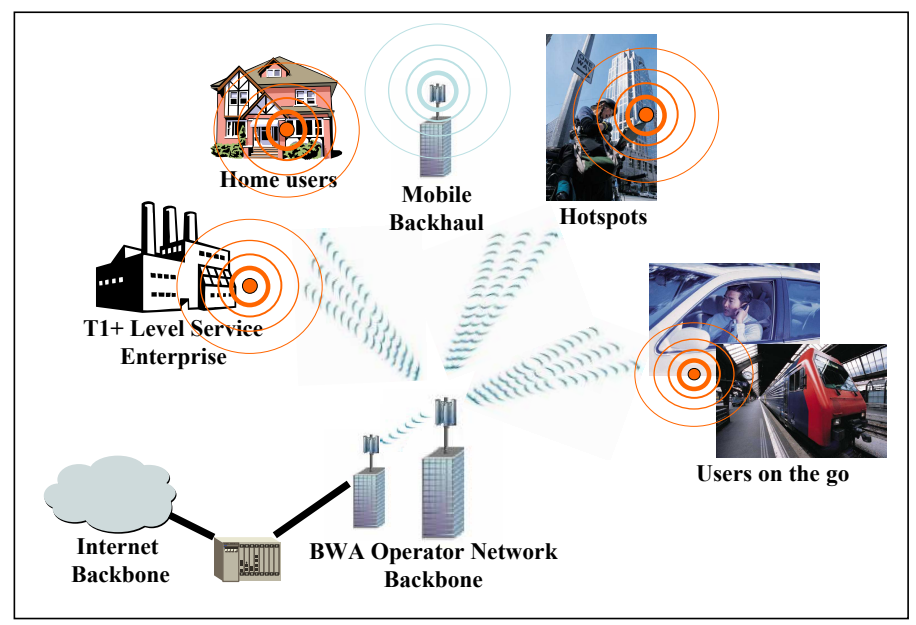

Fig. 1. Illustration of the reference model for interactive OFDMA system

The Evolution of OFDM to OFDMA completely preserves the robustness against multipath propagation and high bandwidth efficiency. However, in case of using multiple antenna for OFDM systems, recovering the transmitted signal is impossible without knowing channel coefficients because the signals from each multipath channel are overlapped. Therefore, channel estimation processing is a major key as recovering the corrupted signal 4. In a typical OFDMA uplink scenario, moreover, multiple access interference (MAI) caused by symbol timing errors destroys the orthogonality among users [5]-77. So, this paper shows our investigations for improving the performance of channel estimation and symbol timing misalignment on OFDMA uplink. At the same time, we provide an algorithm which generates extended-CAZAC (E-CAZAC) sequences to overcome a limitation of the number of transmiting antennas and multipath components to maintain the orthogonality for both uplink and downlink of OFDMA systems. Besides, the Bit error rate (BER) and throughput performance of the interactive OFDMA uplink system for symbol timing misalignment is considered.

The outline the paper is organized as follows. In Section 2, symbol timing error model for OFDMA uplink is described. Section 3 gives the improving channel estimation scheme for both uplink and downlink OFDMA system. Section 4, simulation and numerical results show the mean square error (MSE) of channel estimation and BER performances of OFDMA uplink systems. Finally, the concluding remarks are given Section 5 .

\section{System Model}

In this section we are concerned with the evaluation of the uplink subject to OFDMA system as shown in Fig. 1. The subcarrier frequencies from all users form a set of $N$ orthogonal carriers by appropriate choice of the spacing, as it is 
done in OFDM. As such, each tone in an OFDM symbol is used by a different uplink user. Thus, the users share the same bandwidth at the same time, however orthogonality is achieved by assigning distinct tones to distinct users. These tone assignment schemes consist of interleaved tone assignment scheme that are regularly interleaved across the overall set of $N$ tones and block tone assignment scheme that are assigned to each user with disjoint blocks whose size is $\mathbf{K}_{u}$, where $\mathbf{K}_{u}$ is the number of subcarrier per each user. Usually, The interleaved scheme yields a worse signal to interference ratio (SIR) compared to the block scheme [8]. With the above block scheme in mind, the transmitted signal of the $n$-th subcarrier for the $d$-th user in the time domain can be expressed as

$$
x_{n, d}=\frac{1}{N} \sum_{k=M_{d}(d-1)}^{M_{d} \cdot d-1} X_{k, l} \exp \left[-j 2 \pi \frac{k n}{N}\right]-N_{G} \leq n<N,
$$

where $k$ is a subcarrier index and $M_{d}=\frac{N}{N_{u}}$ is the number of subcarriers assigned to each user. Note that $x_{n, d}$ incorporates the GI of length $N_{G} \leq N$.

The transmitted signal by each user goes through multipath fading channel and the received signal can be expressed as

$$
r_{n}=\frac{1}{N} \sum_{d=1}^{N_{u}} \sum_{k=M_{d}(d-1)}^{M_{d} \cdot d-1} H_{k, d} X_{k, d} \cdot \exp \left[-j 2 \pi n \frac{\left(k-\tau_{d}\right)}{N}\right]+W_{n},
$$

where $W_{n}$ is the zero-mean Gaussian noise (AWGN) with two-sided power spectral density of $N_{0} / 2, \tau_{d}$ is the timing error for the $d$-th user, and $H_{k, d}$ is the discrete frequency response $H_{k, d}=\alpha_{k, d} \cdot \exp \left(j \theta_{k, d}\right)$. In the $H_{k, d}, \alpha_{k, d}$ is the independent identically distributed (i.i.d) Rayleigh random variable and $\theta_{k, d}$ is uniformly distributed random variable over $[-\pi, \pi]$.

Then, the reconstructed FFT output for the $q$-th user's $l$-th subcarrier is given by

$$
R_{l, q}=\frac{1}{N} \sum_{n=0}^{N-1} \sum_{d=1}^{N_{u}} \sum_{k=M_{d}(d-1)}^{M_{d} \cdot d-1} H_{k, d} X_{k, d} \cdot \exp \left[-j 2 \pi n \frac{\left(k-l-\tau_{d}\right)}{N}\right]+W_{n}
$$

where $l \in S_{q}$ and $W_{l}=\sum_{n=0}^{N-1} \exp [j(2 \pi l n / N)]$.

In the equation (3), The MAI caused by symbol timing error $\tau_{d}$ of the multiple access users depends on the transmitted modulated symbol. So, it is difficult for the base station receiver to eliminate or mitigate the MAI. Also, we consider the power of the MAI. Let us assume that the data symbols $X_{k, d}$ which is the $k$-th output signal of the $d$-th user, on different subcarriers of different users are independent of each other, with a zero mean and the same average power $P_{a}$. Without loss of generality, we assume that the channel state is invariant during the observed symbol period. 


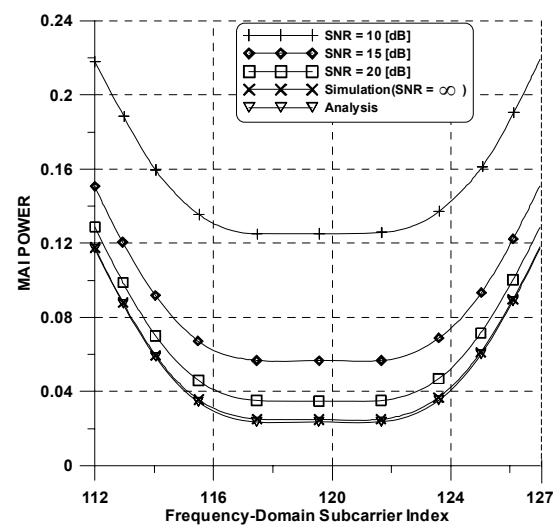

Fig. 2. Interference power plus noise versus frequence domain subcarrier index of the 8-th desired user for $N=256$ and $K_{u}=16$ (16QAM and $\tau_{n, 8}=65$ )

With the above assumptions in mind, we show that the power of the MAI [7] for $k$-th output signal of the $d$-th user is

$$
E|M A I|^{2}=\frac{1}{\pi^{2}} \sum_{n \neq d} \sum_{p=0}^{N-1} E\left|\alpha_{p, n}\right|^{2} P_{a} \times \frac{1-\cos 2 \pi(p-k)\left(\tau_{n, d}-T_{G}\right) / T}{(p-k)^{2}},
$$

where $\tau_{n, d}$ is the symbol timing misalignment of the $n$-th user with respect to the $d$-th user at the receiver, the index $p$ is subcarrier of $n$-th user. And $E\left|\alpha_{p, n}\right|^{2}$ is the average channel gain. $T_{G}$ and $T$ are the cyclic prefix and OFDM symbol duration including guard interval, respectively.

Fig. 2 shows the MAI power for SNR models versus frequency-domain subcarrier index of desired user (the 8-th user) for $N=256$ and $K_{u}=16$. In this figure, we set to the same symbol timing error $\left(\tau_{n, 8}=65\right)$ for all users except the eighth user. The Simulation results were obtained by 16QAM signaling and multipath fading channel with respect to various SNR value, also analysis results were obtain through the average channel gain $E\left|\alpha_{p, n}\right|^{2}=1$. As we can see from this figure, we confirmed what subcarrier between adjacent subbands has higher MAI power. One popular solution to mitigate the MAI is to insert a guard band (GB) among two adjacent subbands and to use the receiver diversity in the base station. The improving performance of the above methods present the Section 4 .

\section{Improving Channel Estimation Using E-CAZAC}

\subsection{CAZAC Sequence}

We introduce OFDMA system with multiple antenna using CAZAC preamble as system model. In section 2 , in order to employ simulation, perfect channel estimation on each user is assumed. However, since channel condition has the 
multipath fading features in practice, we need to estimate the channel coefficients. Also, one of the most dominant factors of the performance degradation is channel estimation error. In order to solve this problem, we achieved the a channel estimation technique using the constant-amplitude zero-autocorrelation (CAZAC) preamble which holds outstanding periodic autocorrelation property is one of the well-known algorithms 9. The CAZAC preamble provide good and rapid signal acquisition performances even for low SNR conditions with 4-phase and 16-symbol-length. However, if the CAZAC preamble is adopted in multiple antennas system, its capability of channel estimation is limited by the number of transmit antennas $\left(N_{t}\right)$ and multipath components $(P)$ to maintain the orthogonality as follows 4 ]

$$
1 \leq P \leq \frac{L}{N_{t}}
$$

where $L$ presents the symbol-length of the CAZAC sequence. In order to overcome such problem, we provide an algorithm which generates extended-CAZAC (E-CAZAC).

\subsection{Proposed Channel Estimation Method}

The E-CAZAC sequences are obtained by zero-padded among the CAZAC sequences in order to maintain orthogonality when multiple antenna are used and multipath component are existed. The follow equation show a structure of the E-CAZAC:

$$
e_{m \cdot L / 4+n}= \begin{cases}j^{m n} & \text { for } n<4 \\ 0 & \text { for } n \geq 4,\end{cases}
$$

where $m \in 0,1,2,3, n \in 0,1, \cdots, L / 4-1$. Remark that $L$ must be times of 4 .

Since such zero paddings do not provide any additional information about the power spectrum, flat power spectrum of CAZAC sequence is inherited. It shows that we can use the autocorrelation of CAZAC sequences. When E-CAZAC sequence is used, we can have more flexible capability of the channel estimation as varied $L$ or $N_{t}$. In addition, One may think other CAZAC sequences with higher phase that better solution for multiple antennas system because all symbols are used for estimation. However, it provides the additional defect because longer sequences with higher phase causes the transmitter complexity and power consumption for transmission or correlation. Therefore, proposed scheme with conventional 4-phase CAZAC sequences can realize a multiple antennas OFDMA system with low hardware complexity and power consumption.

\section{Performance Evaluation and Discussions}

In this section, several simulation results which show the effect of each scheme are provided. To simulate the OFDMA system performance, a flat Rayleigh fading channel on each subcarrier is used and i.i.d fading among different subcarriers is assumed in the simulations. The entire bandwidth (BW) of $20 \mathrm{MHz}$ is divided 


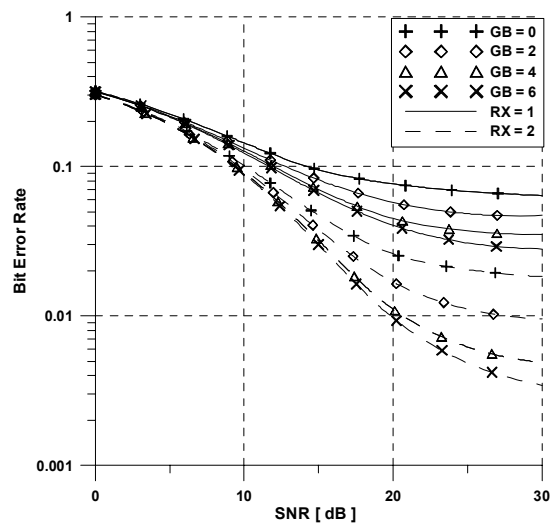

Fig. 3. BER performance of OFDMA uplink systems with respect to the number of GB for $N=256$ and $K_{u}=16$ (16QAM and $\left.\tau_{n, d}=65\right)$

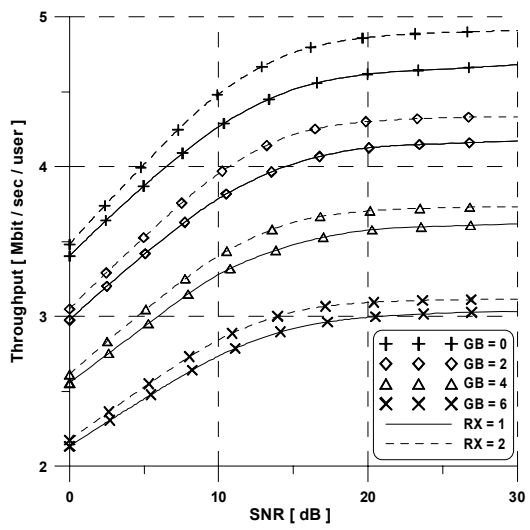

Fig. 4. Throughput performance of OFDMA uplink systems with respect to the number of GB for $N=256$ and $K_{u}=16$ (16QAM, $\tau_{n, d}=65$ and $20 \mathrm{Mhz}$ bandwidth)

into 256 subcarrier. And, the 8-th user is regarded as the desired user. In case of OFDMA uplink channel estimation, we evaluate the performance of proposed ECAZAC sequnce in terms of MSE. Here, we adopt the least square (LS) estimator simulation.

Fig. 3 presents BER performances of OFDMA uplink systems with respect to the number of guard band. A performance enhancement can be observed with increase of the number of GB regardless of the number of receiver antennas. As expected, the BER for the existing MAI give a performance degradation since they destroy the orthogonality among users. However, As the number of guard band increase, throughput is found to decrease as shown in Fig. 4 .

Fig. 5 displays the MSE performances of 32-symbol-length E-CAZAC sequence when 7 paths are presence. In the simulation, we can see that E-CAZAC 


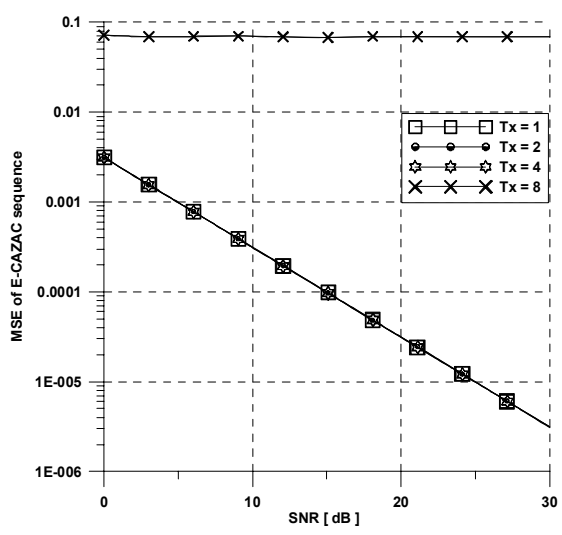

Fig. 5. The MSE performance of OFDMA uplink channel estimation using the ECAZAC for 1, 2, 4 and 8 transmit antennas over 7-path Rayleigh faing channel

sequence performs completely when up to 4 transmit antennas are used as equation (7). In the other case, MSE performaces are very poor because the orthogonality of E-CAZAC sequence is broken. Therefore, we should notice this point. Also, if we want to use 8 transmit antennas, they will estimate the channel condition nicely as increase E-CAZAC sequence length. As expect, the MSE of CAZAC sequence gives the better performance by increasing the number of CAZAC sequence. However, we should use to be properly the symbol-length and number of CAZAC sequences restricted in conformity with the system and channel conditions.

\section{Conclusions}

OFDMA is a promising scheme for providing a ubiquitous environment in wireless channels. However, the both MAI problems and channel estimation problems in case of using the multiple antennas based on OFDMA system caused serious performance degradations. In this paper, we described the MAI reduction schemes in the OFDMA-based interactive wireless system. From the results presented above, we confirmed MAI reduction by using the guard band (GB) and received diversity. At the same time, by the considering system and channel conditions E-CAZAC preamble sequences can provide solutions of the more flexible channel estimation. The presented results are valid for OFDMA systems with reverse link.

\section{Acknowledgement}

This research was supported by the MIC (Ministry of Information and Communication), Korea, under the ITRC (Information Technology Research Center) support program supervised by the IITA (Institute of Information Technology Assessment). 


\section{References}

1. ETSI ETS 301 958, Digital Video Broadcasting (DVB).: Interaction channel for digital terrestrial television (RCT) incorporating multiple access OFDM, ETSI, Tech. Rep., March 2002.

2. IEEE draft standard for local and metropolitan area network-part 16.: Air interface for fixed broadband wireless access systems - medium access control modifications and additional physical layer specifications for 2-11GHz, IEEE LAN MAN Standards Committee, 2002.

3. I. Koffman and V. Roman.: Broadband wireless access solutions based on OFDM access in IEEE802.16, IEEE Commun. Mag., vol. 40, pp. 96-103, April 2002.

4. Dong Jun Cho, Young Hwan You, and Hyoung Kyu Song.: Channel Estimation with Transmitter Diversity for High Rate WPAN Systems, IEICE Trans. Commun., vol. E87- B, no.11 Nov. 2004.

5. Y.-H. You, W.-G. Jeon, J.-W. Wee, Sang-Tae Kim, Intae Hwang, and H.-K. Song.: OFDMA Uplink Performance for Interactive Wireless Broadcasting, IEEE Trans. Broadcast., vol.51, no. 3, pp.383-388, 2005.

6. M. S. El-Tanany, Y. Wu, and L. Hazy.: OFDM uplink for interactive broadband wireless: analysis and simulation in the presence of carrier, clock an timing errors, IEEE Trans. Broadcast., vol. 47, no. 1, pp. 3-19, Mar. 2001.

7. M. Park, K. Ko, H. Yoo, and D. Hong.: Performance analysis of OFDMA uplink systems with symbol timing misalignment, IEEE Commun. Lett., vol. 7, no. 8, pp. 376-378, Aug. 2003.

8. A. Tonello, N. Laurenti, S. Pupolin.: On the effect of time and frequency offsets in the uplink of an asynchronous multi-user DMT OFDMA system, Proc. of Intemutioml Conference on Telecommunications 2000, Acapulco, Mexico, pp.614-618, May 2225.2000 .

9. R. C. Heimiller.: Phase Shift Pulse Codes with Good Periodic Correlation Properties, IRE Trans. Info. Theory IT-6, 254-257 October 1961. 